\title{
Superior ophthalmic vein approach to carotid-cavernous fistulas
}

\section{Technical note}

\author{
Ketan R. Bulsara, M.D., Ram Vasudevan, M.D., and Eren Erdem, M.D. \\ Division of Neurological Surgery, University of Missouri, Columbia, Missouri; and Departments of \\ Neurosurgery and Radiology, University of Arkansas for Medical Sciences, Little Rock, Arkansas
}

\begin{abstract}
Endovascular procedures have revolutionized the treatment of carotid-cavernous fistulas through transarterial and transvenous routes. In some circumstances, however, traditional endovascular approaches may fail. Direct access to the superior ophthalmic vein (SOV) can be used to treat fistulas in this circumstance. The authors discuss the technical aspects of direct exposure of the SOV for access to the cavernous sinus.
\end{abstract}

\section{KEY WORDS • carotid-cavernous fistula • superior ophthalmic vein}

Patients in whom the standard transvenous and transarterial routes for treatment of carotid-cavernous fistulas fail may pose a significant treatment dilemma. Direct cannulation of the SOV may provide a treatment route; however, this technique has rarely been described in detail. ${ }^{1}$ In this article we provide a detailed description of our technique.

\section{DESCRIPTION OF TECHNIQUE}

The procedure can be performed in the radiology suite or in the operating room. Either loupe magnification (minimum $\times 3.5$ ) or an operating microscope can be used. Fluoroscopic guidance is mandatory. After induction of general anesthesia, a femoral sheath is placed.

The incision site (Fig. 1 left) for access to the SOV is prepared using betadine. The incision is made in the nasal aspect of a superior eye fold to maximize the cosmetic outcome. The orbicularis oculi muscle is divided (Fig. 1 center). Meticulous attention to hemostasis with bipolar

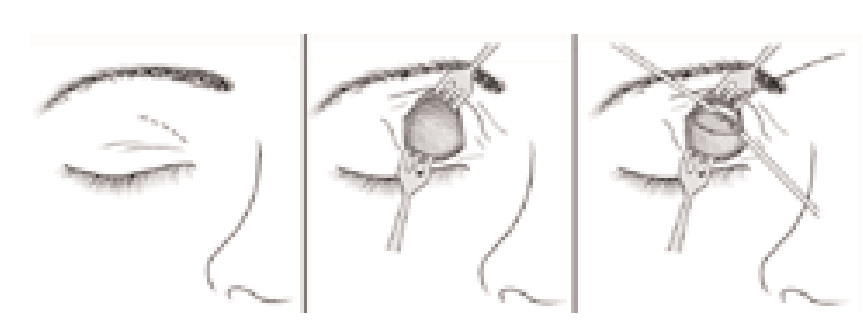

Fig. 1. Drawings showing positioning of the incision (dashed line) in the nasal aspect of the superior eye fold (left), the orbicularis oculi muscle being divided (center), and the isolation of the SOV (right).

Abbreviation used in this paper: $\mathrm{SOV}=$ superior ophthalmic vein. cautery is essential. A microDoppler ultrasonography unit (Mizuho Co., Inc., Nagoya, Japan) assists in identifying the arterialized supratrochlear vein. The orbital septum is opened in the area of the supratrochlear vein, and orbital fat is exposed. The microDoppler unit assists in localizing

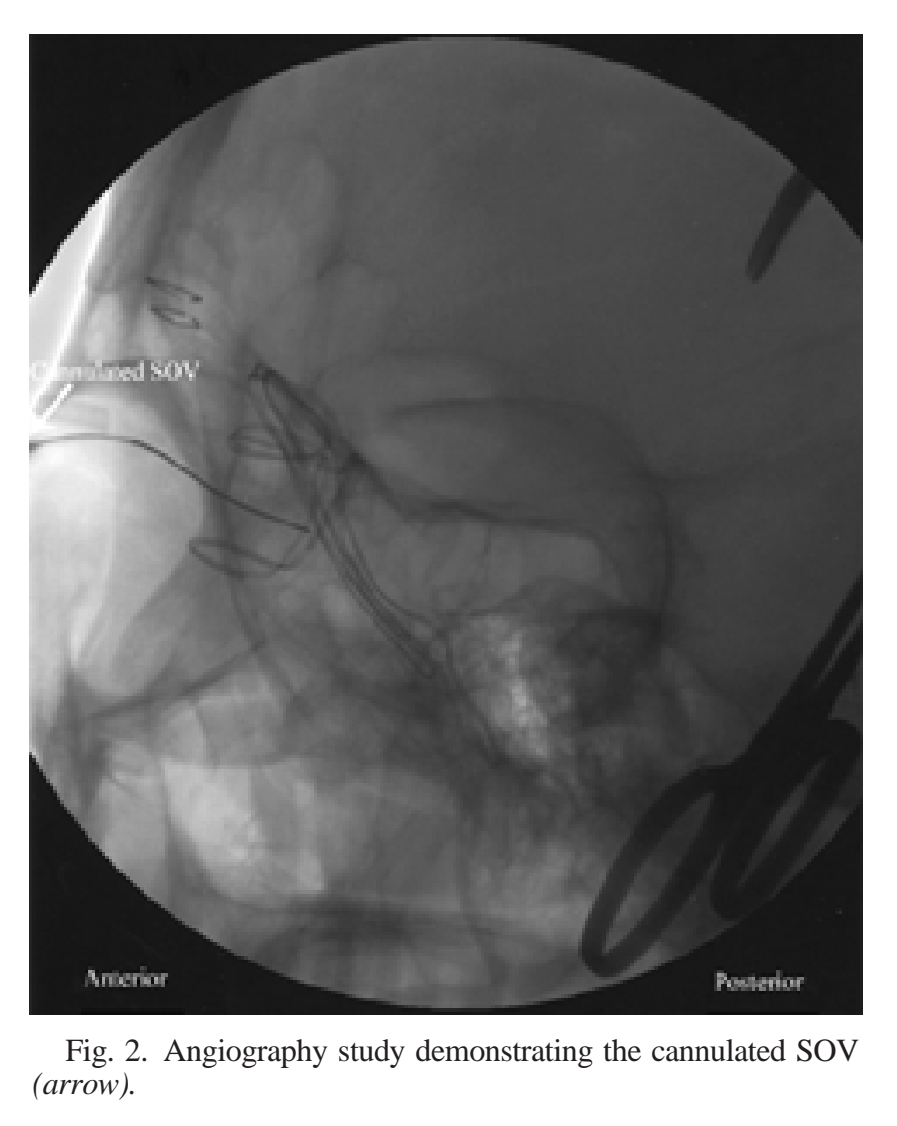


the supratrochlear vein, which can then be followed to the SOV (Fig. 1 right).

The orbital fat surrounding the SOV is carefully removed. Two 2-0 silk ligatures are passed underneath the SOV with a right-angle clamp and separated by approximately $10 \mathrm{~mm}$. Once in place around the vessel, the ligature is passed through a No. 5 French catheter to make a tourniquet. With the ligatures in place, a 20-gauge intravenous needle is used to cannulate the SOV. The needle is held in place with the proximal ligature.

Angiography is performed using a catheter threaded through the femoral artery (Fig. 2). Under fluoroscopic guidance, a catheter is threaded through the SOV to the cavernous sinus. Either platinum coils or a balloon can be delivered using this method.

Once the carotid-cavernous fistula is closed, the SOV is ligated both proximally and distally. Vicryl 5-0 sutures are used to reapproximate the orbicularis oculi muscle.

\section{DISCUSSION}

Most carotid-cavernous fistulas can be treated either through the transarterial or transvenous route. When the standard endovascular routes for access to the fistulas cannot be used, direct cannulation of the SOV is an option. This procedure is relatively simple, although its use has been limited by a lack of adequate descriptions. Collaboration between the neurosurgeon and/or orbital surgeon and interventional radiologist optimizes patient outcome.

\section{Acknowledgment}

We thank Laura Kays for her editorial assistance in the preparation of this manuscript.

\section{Reference}

1. Miller NR, Monsein LH, Debrun GM, et al: Treatment of carotid-cavernous sinus fistulas using a superior ophthalmic vein approach. J Neurosurg 83:838-842, 1995

Manuscript received December 15, 2004.

Accepted in final form January 6, 2005.

Address reprint requests to: Ketan R. Bulsara, M.D., Division of Neurological Surgery, One Hospital Drive, N521, Hospital \& Clinics, Columbia, Missouri 65212. 